\title{
CRESCIMENTO, PRODUÇÃO E QUALIDADE DE FRUTOS DA BANANEIRA 'FIGO CINZA', EM BOTUCATU-SP'
}

\author{
DAYANA PORTES RAMOS ${ }^{2} \&$ SARITA LEONEL ${ }^{3}$
}

\begin{abstract}
RESUMO - A bananeira Figo Cinza é uma cultivar do grupo ABB, subgrupo Figo, que apresenta tolerância ao mal-do-panamá e às sigatokas amarela e negra, demonstrando ser uma cultivar interessante para programas de melhoramento genético. Devido a isso, o presente trabalho teve como objetivo avaliar características do $1^{\circ}$ ciclo da planta, como o crescimento, produção e atributos físico-químicos dos frutos dessa cultivar, no município de Botucatu-SP. Foram avaliadas as seguintes características de crescimento: altura de plantas, circunferência do pseudocaule e número de folhas, medidas na época de emissão da inflorescência; número de dias entre o plantio e o florescimento, número de dias entre o florescimento e a colheita, e número de dias do plantio à colheita. Também foram mensuradas as seguintes características de produção: peso do cacho, número de frutos, peso médio dos frutos, número de pencas e produtividade; na $2^{\mathrm{a}}$ penca foram avaliados peso, número, comprimento e diâmetro dos frutos. Os atributos fisico-químicos analisados foram: textura, $\mathrm{pH}$, acidez titulável, sólidos solúveis, açúcares totais, amido e teor de potássio. Foi utilizado o método das estatísticas descritivas para a caracterização da cultivar, através dos cálculos das médias. Os resultados mostraram que 'Figo Cinza' apresentou porte médio $(2,9 \mathrm{~m})$, ciclo de 420 dias, produtividade média de 12,74 t ha $^{-1}$ e frutos com baixa acidez $(0,06 \%)$.
\end{abstract}

Termos para indexação: Musa sp., desempenho agronômico, características físico-químicas.

\section{GROWTH, YIELD AND QUALITY OF 'FIGO CINZA' BANANA FRUITS IN BOTUCATU-SP}

\begin{abstract}
Figo Cinza' is a cultivar of the genomic group ABB, Figo subgroup, which provides tolerance to Panama and Sigatoka diseases that may be useful in genetic breeding programs. Then, this work aimed to evaluate the plants growth, production and also the physical and chemical characteristics of the fruits from this cultivar in Botucatu city, São Paulo state, Brazil. Some growth characteristics were evaluated such as: plant height, the pseudostem circumference, number of leaves, number of days between planting and flowering, number of days between flowering and harvest and number of days from planting to harvest. These characteristics were measured at the flowering time. The production characteristics such as the bunch weigth, number of fruits, average weigth of the fruits, number of bunches and weight; in the second bunch it was evaluated the weight, the number, the length and the diameter of the fruits. The analyzed physical and chemical characteristics were: firmness, $\mathrm{pH}$, titratable acidity, soluble solids, total sugars, starch and potassium. The used method was descriptive statiscs for the characterization of cultivar by the average calculations. The results showed that 'Figo Cinza' presented a high height $(2.9 \mathrm{~m})$, a cycle of 420 days, yield of $12.74 \mathrm{t} \mathrm{ha}^{-1}$ and the lowest acidity of the fruits $(0.06 \%)$.
\end{abstract}

Index Terms: Musa sp., agronomic characteristics, physical-chemical characteristics

\footnotetext{
${ }^{1}$ (Trabalho 189-08). Recebido em: 21-07-2008. Aceito para publicação em: 04-11-2008.

${ }^{2}$ Mestranda pelo Departamento de Produção Vegetal-Horticultura, Bolsista FAPESP, Faculdade de Ciências Agronômicas/FCA/UNESP

- Cx. P. 237 - 18610-307 - Botucatu-SP.pitchagro@yahoo.com.br

${ }^{3}$ Profa. do Depto. de Produção Vegetal-Horticultura, FCA/UNESP - Cx. P. 237 - 18610-307 - Botucatu-SP. sarinel@fca.unesp.br.
} 


\section{INTRODUÇÃO}

A bananeira Figo Cinza é uma cultivar do grupo $A B B$, subgrupo Figo, que apresenta porte variando entre 3,5 a 4,0 m. O cacho apresenta engaço longo, as pencas variam em número de 6 a 8 e os frutos são relativamente curtos. A casca é grossa, revestida de cera, e o fruto apresenta polpa doce, macia, de cor creme-pálida e saborosa quando perfeitamente madura. É consumida cozida ou frita. Uma característica marcante dos frutos é que eles permanecem com quinas salientes, mesmo no ponto de colheita. Essa cultivar é tolerante à seca, às sigatokas amarela e negra, ao mal-do-panamá e parasitada pela broca. Tem sido utilizado em pequenas áreas, onde as chuvas são escassas (Silva et al., 1999).

Gomes (2002) relata que as cultivares do grupo $\mathrm{ABB}$ não são relevantes economicamente, no entanto demonstram ser interessantes para programas de melhoramento genético, devido à sua tolerância ao mal-do-panamá e às sigatokas amarela e negra.

Gonzaga Neto et al. (1993) concluíram que 'Figo Cinza' se destacou dentro do seu grupo, como promissora na região do Submédio São Francisco, onde apresentou, em média, 3,1 $\mathrm{m}$ de altura, 5 pencas por cacho, cujas pencas apresentaram peso médio de $3,2 \mathrm{~kg}, 14$ frutos, os quais pesaram 320,0 g.

Tendo em vista os poucos dados de pesquisa com essa cultivar de bananeira, que pode mostrar-se promissora em pequenas áreas com problemas de seca e de doenças, o trabalho teve como objetivo avaliar o crescimento das plantas, produção e atributos físico-químicos de frutos de 'Figo Cinza', no município de Botucatu-SP.

\section{MATERIAL E MÉTODOS}

O experimento foi conduzido no pomar experimental do Departamento de Produção Vegetal - Horticultura, da Faculdade de Ciências Agronômicas - UNESP, Câmpus de Botucatu-SP, localizado nas coordenadas geográficas de $22^{\circ} 51^{\prime}$ latitude S, $48^{\circ} 27^{\prime}$ longitude $\mathrm{W}$ e altitude de $810 \mathrm{~m}$. O tipo climático predominante no local é o temperado quente (mesotérmico), com chuvas no verão e seca no inverno (Cwa - Koppen), tendo temperatura média anual de $20,5^{\circ} \mathrm{C}$ e precipitação pluviométrica média anual de $1.533 \mathrm{~mm}$ (Cunha et al., 1999). O solo da área foi classificado como Nitossolo Vermelho, segundo os critérios da Embrapa (1999). Os dados de temperatura e precipitação pluviométrica, medidos durante a condução do experimento, encontram-se na Tabela 1.

As mudas, oriundas de micropropagação, foram transplantadas para o campo com 3 meses de idade, em área previamente preparada, num espaçamento de 2,5 x 2,5 m, com uma área de $6,25 \mathrm{~m}^{2}$ planta $^{-1}$, onde as covas foram abertas com broca, nas dimensões de $60 \mathrm{~cm}$ de diâmetro, $60 \mathrm{~cm}$ de largura e $60 \mathrm{~cm}$ de profundidade.

Durante o primeiro ciclo de produção, as plantas receberam os seguintes tratos culturais: irrigação complementar, baseada em dados de evaporação do Tanque Classe A, com reposição de 100\% da evapotranspiração da cultura, considerando a vazão dos gotejadores de 2,34 $\mathrm{L} \mathrm{hora}^{-1} \mathrm{e}$ um turno de rega a cada 2 dias; controle de plantas daninhas (ROUNDUP $20 \mathrm{~mL} / 200 \mathrm{~L} \mathrm{H}_{2} \mathrm{O}$ ); controle de sigatoka-amarela (Mycosphaerella musicola) 2 vezes ao ano, intercalando FOLICUR 200 CE-0,5 L/ha e MANZATE 800$2 \mathrm{~kg} /$ ha e broca-do-rizoma (Cosmopolites sordidus), em fevereiro de 2007, com Boveril Organic ${ }^{\mathrm{R}}$ (100 iscas/ha - $20 \mathrm{~g}$ ), durante 3 meses; desbaste a cada 2 meses, conduzindo-se 3 plantas; retirada de folhas secas e eliminação do coração, a um espaço de 10 a $12 \mathrm{~cm}$ entre o coração e a última penca.

O delineamento experimental empregado foi o inteiramente casualizado, com 5 repetições e duas plantas úteis por parcela experimental, completamente rodeadas por seis plantas bordadura. Para as análises laboratoriais foram empregados 5 frutos em cada repetição. Foi utilizado o método das estatísticas descritivas, através dos cálculos das médias.

Foram avaliadas as seguintes características de crescimento: altura de plantas, circunferência do pseudocaule a $30 \mathrm{~cm}$ do solo e número de folhas, medidas na época de emissão da inflorescência. Foram calculados o número de dias entre o plantio e o florescimento, número de dias entre o florescimento e a colheita, e número de dias do plantio à colheita. Também foram mensuradas as seguintes características de produção: peso do cacho (peso do engaço+peso das pencas), número e peso das pencas, número de frutos/cacho, peso médio dos frutos (peso das pencas $/ \mathrm{n}^{\circ}$ frutos) e produtividade (peso das pencas $\mathrm{x}$ estande de planta $\mathrm{ha}^{-1}$ ); peso, número, comprimento e diâmetro de frutos da $2^{\mathrm{a}}$ penca. Os atributos fisico-químicos analisados foram: textura, $\mathrm{pH}$, acidez titulável, sólidos solúveis, açúcares totais, amido e teor de potássio.

\section{RESULTADOS E DISCUSSÃO}

A temperatura média durante a realização do experimento (Tabela 1) mostrou-se adequada para o desenvolvimento das bananeiras, não apresentando períodos de temperatura abaixo da mínima para o crescimento das plantas. Segundo Simão (1998), 
temperaturas abaixo de $12^{\circ} \mathrm{C}$ causam paralisação nas atividades da planta. Com relação à precipitação, nos períodos onde a quantidade de chuvas foi insuficiente para suprir as exigências hídricas da cultura, a reposição da água foi realizada através de irrigações, utilizando-se de um sistema de gotejamento. A quantidade aplicada foi baseada na evapotranspiração da cultura.

\section{Características de crescimento de plantas}

Segundo os dados da Tabela 2, a bananeira 'Figo Cinza' apresentou 2,9 $\mathrm{m}$ de altura, pseudocaule com 59,8 cm de circunferência e aproximadamente 13 folhas na época da emissão da inflorescência.

Silva et al. (1999) e Moreira (1999) relataram para 'Figo Cinza' altura variando de 3,5 a 4,0 m. Resultados semelhantes $(3,10 \mathrm{~m})$ foram encontrados por Gonzaga Neto et al. (1993), no Submédio São Francisco. Santos et al. (2006) salientam que, em uma cultivar comercial, é indesejável que a bananeira expresse valores de altura de plantas muito elevados, pois pode dificultar a colheita e provocar o tombamento da planta em decorrência de ventos fortes e ataques de nematóides e broca-das-bananeiras.

A circunferência do pseudocaule está relacionada ao vigor e reflete a capacidade de sustentação do cacho. Os genótipos que apresentam maior diâmetro são menos suscetíveis ao tombamento (Silva \& Alves, 1999). Apesar de não haver relatos de outros trabalhos em relação a essa característica avaliada, verificouse que 'Figo Cinza' apresentou um valor mais baixo $(59,8 \mathrm{~cm})$, quando comparado com outras cultivares de bananeira; como exemplo, Leonel et al. (2004), em Botucatu-SP, observaram para 'Nanicão' uma circunferência de $71,8 \mathrm{~cm}$ a $30 \mathrm{~cm}$ do solo.

De acordo com Soto Ballestero (1992), a presença de mais de oito folhas no florescimento é considerada como suficiente para o desenvolvimento normal do cacho. Champion (1975), ainda, cita que o número de folhas vivas presentes no momento da iniciação floral é regularmente constante, variando de 10 a 12 folhas, corroborando os valores apresentados neste trabalho.

\section{Duração do ciclo das plantas}

De acordo com a Tabela 3, 'Figo Cinza' apresentou 343 dias do plantio ao florescimento, 77 dias do florescimento à colheita e 420 dias do plantio à colheita. Nas condições do Submédio São Francisco, Gonzaga Neto et al. (1993) relataram para 'Figo Cinza' um menor ciclo: 330 dias, devido a essa região apresentar maiores temperaturas e menores altitudes. Quanto maior a temperatura, maior a taxa fotossintética, consequentemente menor o intervalo de dias para o desenvolvimento dos frutos. É de concordância na literatura sobre a cultura que, a cada elevação de $100 \mathrm{~m}$ na altitude, ocorre proporcionalmente um incremento de 30 dias no ciclo vital da planta (Simão, 1998).

O ciclo total apresenta fundamental importância no melhoramento genético da bananeira, pois é um caráter que expressa a precocidade. A redução do número de dias necessários até a colheita representa a antecipação do investimento aplicado (Santos et al., 2006).

\section{Características de produção}

Os resultados da Tabela 4 mostram que o cacho de 'Figo Cinza' pesou $9,16 \mathrm{~kg}$, na qual o engaço (cabo do cacho + ráquis) pesou $1,20 \mathrm{~kg}$, as pencas: $7,96 \mathrm{~kg}$, apresentando produtividade de 12,74 $\mathrm{tha}^{-1}$. O cacho apresentou, em média, os seguintes dados: 55 frutos, 5 pencas, tendo na $2^{\mathrm{a}}$ penca 11 frutos, na qual pesou $1,70 \mathrm{~kg}$, e os mesmos tiveram o comprimento de $18,7 \mathrm{~cm}$, o diâmetro de $41,8 \mathrm{~mm}$ e o peso de $144,59 \mathrm{~g}$.

Moreira (1999) e Silva et al. (1999) relataram valores variando de 6 a 8 pencas, com 12 a 14 frutos e média de $20 \mathrm{~cm}$ de comprimento, sendo esses valores próximos aos relatados neste trabalho.

Sgarbieri et al. (1965) observaram que frutos de bananeira 'Figo', porém não a cultivar Figo cinza, apresentaram valores de peso médio variando de 163,0 (no dia da colheita) a 128,0 g (no $10^{\circ}$ dia de armazenamento), evidenciando que o valor encontrado neste trabalho $(144,59 \mathrm{~g})$ se encontra dentro do intervalo citado.

Gonzaga Neto et al. (1993), no Submédio São Francisco, observaram que cachos de 'Figo Cinza' apresentaram, em média, 5 pencas, contendo 14 frutos cada penca, pesando $3,20 \mathrm{~kg}$ e cada fruto 320 g. Nota-se que os valores são mais elevados, talvez pela melhor adaptação climática da cultivar a regiões mais quentes.

\section{Características de qualidade dos frutos}

No que se refere a alguns atributos de qualidade dos frutos, a 'Figo Cinza' apresentou frutos verdes, com textura média igual a 775,88 $\mathrm{gf} \mathrm{cm}^{-2}$; pH: 6,02; AT: $0,06 \%$; SS: $3,30^{\circ}$ Brix; açúcares totais: 0,15\%; amido: $13,21 \%$ e teor de potássio: $2,98 \mathrm{~g}$ $\mathrm{kg}^{-1}$ (Tabela 5).

Segundo Palmer (1971), o pH da banana verde varia de 5,0 a 5,6 e na banana madura de 4,2 a 4,7 . No entanto, dentro destes limites, podem ocorrer variações nas diferentes cultivares de bananeira. Sgarbieri et al. (1965) observaram para frutos de 'Figo' valores variando de 5,8 (frutos verdes) a 5,0 (frutos maduros), confirmando, também, um $\mathrm{pH}$ elevado para esta cultivar.

De acordo com Bleinroth (1995), Fernandes et al. (1979) e Rossignoli (1983), a acidez em frutos 
de bananeira variou de 0,17 a $0,67 \%$. No entanto, Sgarbieri et al. (1965) observaram maiores valores para 'Figo', variando de 0,19 (frutos verdes) a $0,31 \%$ (frutos maduros). Observa-se que os valores citados são maiores do que os encontrados neste trabalho $(0,06 \%)$, devido terem sido obtidos em regiões diferentes, pois, segundo Silva et al. (2004), o local de avaliação pode interferir na qualidade dos frutos.

Em relação ao teor de sólidos solúveis, Sgarbieri et al. (1965) observaram valores variando de 1,56 (frutos verdes) a 19,40 ${ }^{\circ} \mathrm{Brix}$ (frutos maduros), sendo que o resultado que foi encontrado nesta pesquisa $\left(3,30^{\circ}\right.$ Brix) está dentro dos intervalos citados.

Conforme o relato de Marriot \& Palmer
(1980), os açúcares constituem normalmente 0,5 a 2,0 $\%$ da polpa das bananas verdes, e o amido, de 20 a 25

$\%$. Sgarbieri et al. (1965) observaram que os valores de amido variaram de 24,30 (no dia da colheita) a $16,82 \%$ (no $10^{\circ}$ dia de armazenamento).

Bleinroth (1990) cita que os sais minerais apresentam pequena variação durante a maturação da fruta, sendo seus teores na banana verde um pouco maiores do que no fruto maduro. A banana é considerada como uma importante fonte de potássio na alimentação e, de acordo com o mesmo autor, a concentração desse mineral na fruta madura é de 350-400 mg $100 \mathrm{~g}^{-1}$ de matéria seca, o que corresponde a $0,35-0,40 \%$. Os valores obtidos no presente trabalho $(0,30 \%)$ estão próximos aos relatados pelo autor citado.

TABELA 1 - Médias mensais das temperaturas máxima, média e mínima $\left({ }^{\circ} \mathrm{C}\right)$, e precipitação pluviométrica (mm) medidas durante os anos de 2006 e 2007. Fonte: Departamento de Recursos Naturais - FCA/UNESP, Botucatu-SP.

\begin{tabular}{lcccccccc}
\hline \multirow{2}{*}{ Meses } & \multicolumn{2}{c}{$\begin{array}{l}\text { Temperatura } \\
\text { máxima }\left({ }^{\circ} \mathbf{C}\right)\end{array}$} & \multicolumn{2}{c}{$\begin{array}{l}\text { Temperatura } \\
\text { média }\left({ }^{\circ} \mathbf{C}\right)\end{array}$} & \multicolumn{2}{c}{$\begin{array}{l}\text { Temperatura } \\
\text { mínima }\left({ }^{\circ} \mathbf{C}\right)\end{array}$} & \multicolumn{2}{c}{$\begin{array}{l}\text { Preciptação } \\
\text { pluviométrica } \\
(\mathbf{m m})\end{array}$} \\
\cline { 2 - 10 } & 2006 & 2007 & 2006 & 2007 & 2006 & 2007 & 2006 & 2007 \\
\hline Janeiro & 28,6 & 26,9 & 21,0 & 23,0 & 19,5 & 19,2 & 172,0 & 410,8 \\
Fevereiro & 28,1 & 29,3 & 21,8 & 24,1 & 19,2 & 18,9 & 262,7 & 108,9 \\
Março & 28,1 & 30,2 & 21,7 & 24,8 & 19,2 & 19,4 & 211,2 & 49,0 \\
Abril & 25,8 & 27,6 & 20,3 & 22,8 & 16,2 & 18,0 & 60,9 & 40,3 \\
Maio & 22,7 & 23,3 & 15,2 & 18,5 & 12,8 & 13,6 & 8,7 & 42,1 \\
Junho & 23,4 & 25,2 & 18,2 & 19,8 & 13,1 & 14,4 & 15,4 & 23,4 \\
Julho & 24,4 & 22,7 & 17,9 & 17,9 & 13,9 & 13,0 & 0,9 & 172,6 \\
Agosto & 26,8 & 25,6 & 20,6 & 20,4 & 14,3 & 15,1 & 0,2 & 0 \\
Setembro & 25,5 & 28,9 & 19,9 & 23,1 & 14,2 & 17,3 & 1,5 & 10 \\
Outubro & 27,4 & 30,2 & 16,1 & 23,9 & 21,7 & 17,4 & 3,1 & 77,7 \\
Novembro & 28,1 & 26,5 & 17,2 & 22,0 & 20,9 & 17,5 & 2,8 & 177 \\
Dezembro & 28,3 & 28,1 & 18,9 & 22,7 & 23,6 & 18,3 & 7,3 & 180,6 \\
\hline Média & 26,4 & 27,0 & 19,1 & 21,9 & 17,4 & 16,8 & 62,2 & 107,7 \\
\hline
\end{tabular}

TABELA 2 - Valores médios de altura de plantas, circunferência do pseudocaule e número de folhas por planta, da cultivar de bananeira Figo Cinza, em Botucatu-SP (jan 2006 a jan 2008).

\begin{tabular}{ccc}
\hline $\begin{array}{c}\text { Altura de plantas } \\
(\mathbf{m})\end{array}$ & $\begin{array}{c}\text { Circunferêncía do pseudocaule } \\
(\mathbf{c m})\end{array}$ & Número de folhas por planta \\
\hline 2,9 & 59,8 & 12,8 \\
\hline
\end{tabular}

TABELA 3 - Valores médios de número de dias do plantio ao florescimento, número de dias entre o florescimento e a colheita e número de dias do plantio à colheita (ciclo), da cultivar de bananeira Figo Cinza, em Botucatu-SP (jan 2006 a jan 2008).

\begin{tabular}{ccc}
\hline $\begin{array}{c}\mathbf{N}^{0} \text { de dias do plantio ao } \\
\text { florescimento }\end{array}$ & $\begin{array}{c}\mathbf{N}^{0} \text { de dias do florescimento à } \\
\text { colheita }\end{array}$ & $\begin{array}{c}\mathbf{N}^{0} \text { de dias do plantio à colheita } \\
\text { (ciclo) }\end{array}$ \\
\hline 343 & 77 & 420 \\
\hline
\end{tabular}


TABELA 4 - Valores médios do peso das pencas (PP), peso do engaço (PENG), peso do cacho (PC), número de frutos por cacho $\left(\mathrm{N}^{\circ} \mathrm{F}\right)$, peso do fruto $(\mathrm{PF})$, produtividade (PROD), número de pencas por cacho $\left(\mathrm{N}^{\circ} \mathrm{P}\right)$, peso $\left(\mathrm{P} 2^{\circ} \mathrm{P}\right)$, número de frutos $\left(\mathrm{F} 2^{\circ} \mathrm{P}\right)$, comprimento $(\mathrm{CF})$ e diâmetro de frutos $(\mathrm{DF})$ na $2^{\mathrm{a}}$ penca, da cultivar de bananeira Figo Cinza, em Botucatu-SP (jan 2006 a jan 2008).

\begin{tabular}{ccccccccccc}
\hline $\begin{array}{c}\text { PP } \\
(\mathrm{kg})\end{array}$ & $\begin{array}{c}\text { PENG } \\
(\mathrm{kg})\end{array}$ & $\begin{array}{c}\mathrm{PC} \\
(\mathrm{kg})\end{array}$ & $\mathrm{N}^{\circ} \mathrm{F}$ & $\begin{array}{c}\mathrm{PF} \\
(\mathrm{g})\end{array}$ & $\begin{array}{c}\text { PROD } \\
\left(\mathrm{tha}^{-1}\right)\end{array}$ & $\mathrm{N}^{\circ} \mathrm{P}$ & $\begin{array}{c}\mathrm{P}^{\circ} \mathrm{P} \\
(\mathrm{kg})\end{array}$ & $\mathrm{F}^{\circ} \mathrm{P}$ & $\begin{array}{c}\mathrm{CF} \\
(\mathrm{cm})\end{array}$ & $\begin{array}{c}\mathrm{DF} \\
(\mathrm{mm})\end{array}$ \\
\hline 7,96 & 1,20 & 9,16 & 55 & 144,59 & 12,74 & 5 & 1,70 & 11 & 18,7 & 41,8 \\
\hline
\end{tabular}

TABELA 5 - Valores médios de textura, pH, acidez titulável (AT), sólidos solúveis (SS), açúcares totais (ACT), amido e teor de potássio $(\mathrm{K})$ dos frutos da cultivar de bananeira Figo Cinza, em Botucatu-SP.

\begin{tabular}{ccccccc}
\hline $\begin{array}{c}\text { Textura } \\
\left(\mathrm{gf} \mathrm{cm}^{-2}\right)\end{array}$ & $\mathrm{pH}$ & $\begin{array}{c}\text { AT } \\
(\%)\end{array}$ & $\begin{array}{c}\text { SS } \\
\left({ }^{\circ} \text { Brix }\right)\end{array}$ & $\begin{array}{c}\text { ACT } \\
(\%)\end{array}$ & $\begin{array}{c}\text { Amido } \\
(\%)\end{array}$ & $\begin{array}{c}\mathrm{K} \\
\left(\mathrm{g} \mathrm{kg}^{-1}\right)\end{array}$ \\
\hline 775,88 & 6,02 & 0,06 & 3,30 & 0,15 & 13,21 & 2,98 \\
\hline
\end{tabular}

\section{CONCLUSÃO}

Os resultados mostraram que 'Figo Cinza' apresentou porte médio ( $2,9 \mathrm{~m})$, um ciclo de 420 dias, produtividade média de $12,74 \mathrm{tha}^{-1}$, frutos com baixa acidez $(0,06 \%)$, textura média igual a $775,88 \mathrm{gf} \mathrm{cm}^{-2}$, pH: 6,02, acidez titulável: $0,06 \%$, sólidos solúveis: $3,30^{\circ}$ Brix, açúcares totais: $0,15 \%$, amido: $13,21 \%$ e teor de potássio: $2,98 \mathrm{~g} \mathrm{~kg}^{-1}$.

\section{AGRADECIMENTO}

À Fundação de Amparo à Pesquisa do Estado de São Paulo - FAPESP, pelo suporte financeiro que viabilizou a execução deste trabalho.

\section{REFERÊNCIAS}

BLEINROTH, E. W. Matéria-prima. In: ITAL. Banana: cultura, matéria-prima, processamento e aspectos econômicos. 2. ed.. Campinas, 1990. p. 163-96. (Série frutas tropicais, 3).

BLEINROTH, E. W. Matéria-prima. In: ITAL.. Banana: cultura, matéria-prima, processamento e aspectos econômicos. 2. ed. Campinas: ITAL, 1995. cap. 2, p. 133-196.

CHAMPION, J. El plátano: técnicas agrícolas y producciones tropicales. Barcelona: Blume, 1975. p. .3-55.

CUNHA, A.R.; KLOSOWSKI, E.S.; GALVANI, E.; SCOBEDO, J.F.; MARTINS, D. classificação climática para o município de Botucatu-SP, segundo
Köppen. In: SIMPÓSIO EM ENERGIA NA AGRICULTURA, 1., 1999, Botucatu. Anais... Botucatu: Faculdade de Ciências Agronômicas, 1999. p. 490491.

EMBRAPA. Centro Nacional de Pesquisa de Solos. Sistema brasileiro de classificação de solos. Rio de Janeiro, 1999. 412 p.

FERNANDES, K. M.; CARVALHO, V. D. de; CALVIDAL, J. Physical changes during ripening of silver bananas. Journal of Food Science, Chicago, v. 44, n. 4, p. 1254-1255, 1979.

GOMES, G. A. C. Divergência genética entre cultivares e certificação genética e fitossanitária de matrizes de bananeira. 2002. $124 \mathrm{f}$. Tese (Doutorado em Fitotecnia)- Universidade Federal de Lavras, Lavras, 2002.

GONZAGA NETO, L.; SOARES, J. M.; CRISTO, A. S.; NASCIMENTO. Avaliação de cultivares de bananeira na região do Submédio São Francisco: primeiro ciclo de produção. Revista Brasileira de Fruticultura, Jaboticabal,, v. 15, n. 1, p. 21-25, 1993.

LEONEL, S.; GOMES, E. M.; PEDROSO, C. J. Desempenho agronômico de bananeiras micropropagadas em Botucatu-SP. Revista Brasileira de Fruticultura, Jaboticabal, v. 26, n. 2, p. 245-248, 2004.

MARRIOT, J.; PALMER, J. K. Bananas: physiology and biochemistry of storage and ripening for optimum quality. CRC Critical Review in Food 
Science and Nutrition, Boca Raton, v. 13, n. 1, p. 41,1980

MOREIRA, R. S. Banana: teoria e prática de cultivo. 2. ed. São Paulo: Fundação Cargill, 1999. CD-ROM.

PALMER, J. K. The banana. In: HUME, A. C. (Ed.). The biochemistry of fruits and their products. London: Academic Press, 1971. v. 2, p. 65-101.

ROSSIGNOLI, P. A. Atmosfera modificada por filmes de polietileno de baixa densidade com diferentes espessuras para conservação de banana 'Prata' em condições ambiente. 1983. 81 f. Dissertação (Mestrado em Ciência dos Alimentos) Escola Superior de Agricultura de Lavras, Lavras, 1983.

SANTOS, S. C. et al. Caracterização morfológica e avaliação de cultivares de bananeira resistentes a sigatoka-negra (Mycosphaerella fijiensis Morelet) no sudoeste goiano. Revista Brasileira de Fruticultura, Jaboticabal, v. 28, n. 3, p. 449-453, 2006.

SGARBIERI, V. C.; HEC, M.; LEONARD, S. J. Estudo bioquímico de algumas variedades de bana- na cultivadas no Brasil. Coletânea do Instituto de Tecnologia de Alimentos, Campinas, v. 1, n. 2, p. 527-558, 1965/66.

SANTOS, S.C.; CARNEIRO, L.C.; SILVEIRA NETO, A.N.; Paniago Júnior, E.; Freitas, H.G.; Peixoto, C.N. Cultivares. In: ALVES, E. J. (Org.). A cultura da banana: aspectos técnicos, socioeconômicos e agroindustriais. 2. ed. Brasília: EMBRAPA, SPI, 1999. p. 103.

SILVA, S. de O. e; ALVES, E. J. Melhoramento genético e novas cultivares de bananeira. Informe Agropecuário, Belo Horizonte, v. 20, n. 196, p. 91-96, 1999.

SILVA, S. de O. e; SANTOS-SEREJO, J. A. dos; CORDEIRO, Z. J. M. Variedades. In: BORGES, A. L.; SOUZA, L. da S. (Eds.). O cultivo da bananeira. Cruz das Almas: EMBRAPA Mandioca e Fruticultura, 2004. p. 45-58.

SIMÃO, S. Bananeira. In: SIMÃO, S. Tratado de fruticultura. Piracicaba: FEALQ, 1998. p. $327-$ 381 .

SOTO BALLESTERO, M. Bananas: cultivo y comercialización. 2. ed. San José: Litografia e Imprensa, 1992. 674 p. 